\title{
PERFIL DOS CANDIDATOS À LISTA DE TRANSPLANTE RENAL COM DOADOR FALECIDO EM UM CENTRO DE TERAPIA RENAL SUBSTITUTIVA NO BRASIL
}

\author{
Profile of candidates for the transplant waiting list of deceased kidney donor at a center \\ providing renal replacement therapy in Brazil
}

André Barreto Pereira', Maria Goretti Moreira Guimarães Penido², Milton Soares Campos Neto ${ }^{3}$, Gustavo Mário Capanema Silva ${ }^{3}$

\begin{abstract}
RESUMO
Introdução: A literatura não oferece consenso quanto às indicações e contra-indicações para inclusão em lista para transplante renal com doador falecido nos centros de terapia renal substitutiva. Objeivos: avaliar o perfil demográfico, as razões das indicações ou não para inclusão em lista de transplante renal doador falecido e a enfermidade primária dos pacientes em terapia renal substitutiva desse centro. Métodos: Foi analisado o banco de dados de pacientes em terapia renal substitutiva quanto aos dados demográficos, às enfermidades primárias eàs indicações e contra-indicações para inclusão em lista de transplante renal doador falecido. Foram divididos em três grupos: G1 (aptos ao transplante), G2 (não aptos) e G 3 (avaliação). Resultados: de 408 pacientes, 127 estavam no G1, eram 54\% masculinos, idade média 43 anos (15-65), 41\% tinham glomerulonef rite crônica, 12\% nef roesclerose hipertensiva, 10\% nefrite túbulo intersticial crônica, $6 \%$ diabetes melitus e $6 \%$ nefropatia de refluxo. No G2 havia 166 pacientes, 46\% masculinos, idade média 62 anos (25-88), 30\% tinham diabetes melitus, 15\% glomerulonef rite crônica e 14\% nef roesclerose hipertensiva, 11\% doença ateroembólica. No G3 havia 115 pacientes, $62 \%$ masculinos, idade média 49 anos (16-65), 42\% com diabetes melitus, 15\% glomerulonefrite crônica, 8\% nefrite túbulo intersticial crônica, $4 \%$ doença ateroembólica e $48 \%$ necessitavam de avaliação cardiológica. A s principais razões para exclusão no $\mathrm{G} 2$ foram: idade superior a 65 anos e recusa ao Transplante. Condusões: um percentual esperado de pacientes desse centro encontrava-se apto à inclusão em lista de transplante renal doador falecido (31\%) e as maiores contra-indicações foram idade avançada e recusa.
\end{abstract}

Descritores: diálise, insuficiência renal crônica e lista de espera para transplante renal

\footnotetext{
Grau acadêmico

1. Médico nefrologista mestrando

2. Médica nefrologista pediatra professora doutora

3. Médico nefrologista
}

Instituições:

Centro de Nefrologia Santa Casa de Belo Horizonte, Belo Horizonte, MG, Brasil Hospital das Clínicas da Universidade Federal de Minas Gerais

Correspondência:

André Barreto Pereira

Centro de Nefrologia da Santa Casa de Belo Horizonte

Rua Piauí, 420, Bairro Santa Efigênia, Belo Horizonte, MG, Brasil

CEP: 30150320

Telefone: 553132388169

Email: andrebarper@yahoo.com.br

Recebido em: 07/08/2005

Aceito em: 30/09/2005

\section{INTRODUÇÃO:}

A avaliação clínica, laboratorial e de imagem de pacientes candidatos a transplante renal com doador falecido está cada vez mais detalhada e a indicação, mais cuidadosa. 0 paciente renal crônico apresenta particularidades na evolução da doença cardiovascular, merecendo uma avaliação criteriosa e delicada. $\mathrm{Na}$ população incidente em hemodiálise sem sintomas coronarianos, 53,3\% têm estenose significante de artéria coronariana, sendo 83,3\% dos diabéticos. ${ }^{1,2} \mathrm{~A}$ pesar das características da população incidente em diálise serem diferentes daquela em lista de espera de rim doador falecido, estes dados demonstram a grande probabilidade de doença coronariana oculta nos candidatos a transplante renal. ${ }^{1,2} \mathrm{~A}$ literatura não oferece consenso quanto à prevalência das indicações e contraindicações para inclusão em lista nos centros de terapia renal substitutiva (TRS). Segundo a Sociedade B rasilei ra de N ef rologia, existem, aproximadamente, 65.000 pacientes com doença renal crônica (DRC) em tratamento dialítico no Brasil em 2005; um aumento de 6.000 pessoas em relação a 2004. ${ }^{3} \mathrm{~A}$ pesar de não haver dados do número de pacientes potenciais receptores de transplante renal, estima-se que, aproximadamente, um terço daqueles em tratamento dialítico compõe este número. No Brasil, em 2004, segundo a A ssociação B rasilei ra de Transplantes de Ó rgãos, foram realizados 3.332 transplantes renais, ou seja, aproximadamente $15 \%$ dos candidatos na lista de espera de transplante renal doador 
falecido. Em M inas Gerais (M G) ocorreram 384 transplantes em 2004, compreendendo aproximadamente 10\% (3815 pacientes da lista de espera de transplante renal doador cadáver em dezembro de 2004). Destes, 185 de doador vivo. ${ }^{4} 0$ transplante renal é a melhor modal idade de tratamento para o paciente renal crônico dependente de diálise, uma vez considerado o custo do procedimento e a qualidade de vida. Com o progressivo aumento da população dialítica, torna-se de fundamental importância possibilitar ao paciente 0 transplante renal como uma perspectiva de tratamento. Os dados epidemiológicos de cada centro dialítico provavelmente variam de acordo com a região onde o centro se situa, mas é de importância o conhecimento disto. Considerando o número significativo de pacientes em tratamento dialítico no Centro de N efrologia da Santa Casa de B el o Horizonte (C N SCBH), avaliou-se o perfil demográfico, as razões das indicações ou não para inclusão em lista e a enfermidade primária dos indivíduos em TRS nesse centro.

\section{Métodos}

Em fevereiro de 2005, analisou-se no banco de dados local do referido centro, segundo protocolo estabelecido para este fim os pacientes em TRS quanto ao sexo, faixa etária, doença primária, sorologia para hepatite B (HBV) e hepatite C (HCV), tempo de diálise, situação na lista de espera de transplante renal doador falecido e as razões das indicações ou não para inclusão nesta. A mortalidade dos grupos foi avaliada em um ano.

Os pacientes foram divididos em três grupos: G1 (aptos ao transplante), G2 (não aptos) e G3 (em investigação). A s variáveis acima relatadas foram analisadas em cada grupo. A etiologia primária foi diagnosticada principalmente baseada na anamnese do paciente (glomerulonefrite crônica, nefrite tubulointersticial crônica, nefropatia do refluxo, etc), com exceção daqueles casos em que o exame laboratorial ou de imagem é fundamental para o diagnóstico (mieloma, doença renal policística autossômica dominante, etc).

A análise estatística foi realizada utilizando o teste não paramétrico de $\mathrm{K}$ ruskal-Wallis, teste de $\mathrm{M}$ ann-W hitney com valor $\mathrm{p}$ corrigido, o teste qui-quadrado e o teste exato de Fisher, todos com um nível de $5 \%$ de significância. 0 estudo foi aprovado pelo Comitê de Ética em Pesquisa (CEP) local.

\section{Resultados}

Foram avaliados 408 pacientes. Entre os três grupos houve diferença estatísticamente significativa entre idade $(G 1: 42,70 \pm 10,91$, $\mathrm{G} 2: 62,10 \pm 15,32, \mathrm{G} 3: 48,68 \pm 12,17$ anos, $p=0,000)$, mortalidade em um ano ( $G 1: 4,72 \%, G 2: 22,89 \%, G 3: 10,43 \% p=0,000)$ e tempo de diálise (G1:1479, G2:1469, G3:1035 p=0,000) com exceção na idade entre os aptos e não aptos $(p=0,198)$. Não houve diferença entre sexo e entre sorologia para hepatite $\mathrm{C}$ nos grupos.

0 grupo I (aptos) compreendia 127 (31\%) indivíduos. Destes, $54 \%$ eram masculinos, $41 \%$ tinham glomerulonefrite crônica (GNC), 12\% nefroesclerose hipertensiva ( $\mathrm{NH}$ ), 10\% nefrite túbulo intersticial crônica (NTIC), 6\% diabetes mellitus (DM) e 6\% nefropatia de refluxo (NR). No G2 (não aptos) havia 166 pacientes (40\%), 48\% masculinos, idade média 62 a (25-88), 30\% tinham DM , 15\% (GNC) e 14\% N H, 11\% doença ateroembólica (DA E ), 8\%
NTIC. No G3 (em investigação) havia 115 pacientes (28\%), 62\% masculinos, $42 \%$ com DM , 15\% GN C, $8 \%$ NTIC, 4\% DAE, $10 \%$ NR. N este grupo 46\% necessitavam de avaliação cardiológica, 17\% necessitavam de avaliação urológica e $11 \%$ estavam em avaliação pelo grupo de hepatites.

Houve diferença estatisticamente significativa entre os três grupos $(\mathrm{G} 1, \mathrm{G} 2, \mathrm{G} 3)$ em relação às etiologias de DM $(\mathrm{G} 1: 5,51 \%, \mathrm{G} 2: 29,76 \%$, G3:41,74\% p:0,000), DAE (G1:3,15\%,G2:11,31\% , G3:4,35\% p:0,009), NH (G1:11,8\%,G2:14,29\%, G3:3,48\% p:0,011), NR (G1:6,3\%, G2:2,38\%, G3:9,57\% p:0,035), GNC (G1:40,94\%, $\mathrm{G} 2: 15,48 \%, \mathrm{G} 3: 14,78 \%$ p:0,000) e nefropatia crônica de enxerto (NCE) $(G 1: 3,15 \%, G 2: 0 \%, G 3: 0,87 \%$ p:0,048), e à mortalidade (tabela 1).

A s principais razões para exclusão no $\mathrm{G} 2$ foram: idade maior $65 a$ $(54 \%)$, recusa do paciente ao transplante $(14 \%)$, contra-indicação cardiológica ( $8 \%)$, recusa à biópsia hepática $(5 \%)$, pacientes com hepatite $\mathrm{B}$ ou hepatite $\mathrm{C}$ que necessitavam deste procedimento para adequada avaliação (tabela 2).

\section{Discussão}

Como relatado previamente na introdução, é esperado que um terço daqueles em tratamento dialítico esteja apto para o transplante renal, como foi achado neste estudo. 0 centro de diálise anal isado situa-se dentro de um complexo hospitalar onde a grande maioria dos pacientes é proveniente do Sistema Ú nico de Saúde (SUS), encaminhada de outros serviços de saúde de menor porte da região metropolitana e do interior do estado de M inas Gerais, sem estrutura para um atendimento nefrológico. E ste resultado da prevalência de pacientes na lista de transplante acompanha aqueles publicados em outros trabal hos. ${ }^{5}$

Houve diferença estatísticamente significativa na mortalidade entre os grupos, sendo maior no G2 (não aptos), seguido pelo G3 (em investigação) e G1 (aptos), como o esperado. A proporção de pacientes do sexo feminino e masculino é semelhante dentro dos grupos. No grupo "em investigação", a maior pendência é a avaliação cardiovascular (46\%), seguida pela urológica (17\%). É de conhecimento a gravidade cardiovascular do paciente renal crônico, sendo aquela doença a maior causa de óbito. A lém do risco peroperatório, existem outros motivos para uma adequada aval iação desta população. A proximadamente metade das mortes em 30 dias pós-transplante é devida a eventos cardíacos. ${ }^{1} \mathrm{~A}$ necessidade de imunossupressores por longo prazo pode agravar a doença aterosclerótica. U ma vez que rins de doadores falecidos ou vivos são de uma preciosidade imensurável e a maior causa de perda de enxerto é a morte por evento cardiovascular, sua utilização deve ser bem analisada. Torna-se prudente, portanto, uma adequada avaliação destes para o procedimento cirúrgico do transplante, principalmente naqueles diabéticos. ${ }^{1}$ Esta necessidade tornou-se uma exigência do órgão regulamentador do transplante neste estado (M G -Transplante), para todo paciente maior que 65 anos, e diabético maior que 45 anos encaminhados para inclusão na lista de transplante renal com doador falecido. A avaliação urológica é obrigatória para aqueles masculinos maiores que 45 anos.

A maior causa de contra indicação de inclusão em lista de transplante era a idade do paciente, seguida pela recusa do paciente em ser inscrito. Infel izmente, a demanda de órgãos para 


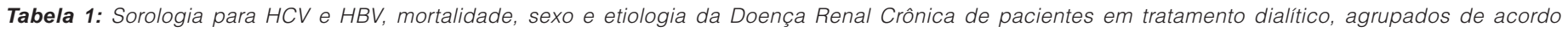
com a situação na lista de transplante renal com doador falecido.

\begin{tabular}{|c|c|c|c|c|c|c|c|}
\hline \multirow[b]{2}{*}{ ETIOLOGIA DE DRC } & \multicolumn{2}{|c|}{$\begin{array}{l}\text { Aptos } \\
n=127\end{array}$} & \multicolumn{2}{|c|}{$\begin{array}{c}\text { não aptos } \\
n=166\end{array}$} & \multicolumn{3}{|c|}{$\begin{array}{c}\text { em investigação } \\
n=115\end{array}$} \\
\hline & $n$ & $\%$ & $n$ & $\%$ & $n$ & $\%$ & Valor $p$ \\
\hline nefropatia diabética & 7 & 5,51 & 50 & 29,76 & 48 & 41,74 & $0,000^{*}$ \\
\hline doença ateroembólica & 4 & 3,15 & 19 & 11,31 & 5 & 4,35 & $0,009^{*}$ \\
\hline GNC & 52 & 40,94 & 26 & 15,48 & 17 & 14,78 & $0,000^{*}$ \\
\hline NTIC & 12 & 9,45 & 13 & 7,74 & 8 & 6,96 & 0,768 \\
\hline síndrome de alport & 5 & 3,94 & 1 & 0,60 & 1 & 0,87 & 0,066 \\
\hline DRPAD & 4 & 3,15 & 7 & 4,17 & 4 & 3,48 & 0,883 \\
\hline indeterminado & 3 & 2,36 & 1 & 0,6 & 4 & 3,48 & 0,215 \\
\hline nefrite lúpica & 3 & 2,36 & 3 & 1,79 & 0 & 0 & 0,280 \\
\hline nefroesclerose hipertensiva & 15 & 11,81 & 24 & 14,29 & 4 & 3,48 & $0,011^{*}$ \\
\hline nefropatia de refluxo & 8 & 6,30 & 4 & 2,38 & 11 & 9,57 & $0,035^{*}$ \\
\hline nefropatia crônica de enxerto & 4 & 3,15 & 0 & 0 & 1 & 0,87 & $0,048^{*}$ \\
\hline neoplasia & 1 & 0,79 & 3 & 1,79 & 0 & 0 & 0,308 \\
\hline vasculite & 2 & 1,57 & 2 & 1,19 & 2 & 1,74 & 0,929 \\
\hline outras & 7 & 5,51 & 13 & 7,74 & 10 & 8,70 & 0,609 \\
\hline Sexo & 69 & 62,00 & 79 & 48,00 & 62 & 54,00 & 0,429 \\
\hline Hepatite C & 14 & 12,17 & 24 & 14,46 & 7 & 5,51 & 0,088 \\
\hline Hepatite B & 7 & 6,09 & 3 & 1,81 & - & - & - \\
\hline \multirow[t]{2}{*}{ óbitos } & 6 & 4,72 & 38 & 22,89 & 12 & 10,43 & $0,000^{*}$ \\
\hline & média & d.p. & média & d.p. & média & d.p. & \\
\hline idade & 42,70 & 10,91 & 62,10 & 15,32 & 48,68 & 12,17 & $0,000^{*}$ \\
\hline
\end{tabular}

Tabela 2: Razões de não aptidão para inclusão na lista de transplante renal com doador falecido

\begin{tabular}{lcc}
\hline razões para não inclusão em lista & $n$ & $\%$ \\
\hline idade (>70) & 59 & 35,54 \\
idade (65-70) & 31 & 18,67 \\
recusa & 24 & 14,46 \\
cardiopatia & 13 & 7,83 \\
recusa a biópsia hepática & 8 & 4,82 \\
não aderência & 7 & 4,22 \\
obesidade & 5 & 3,01 \\
uropatia & 4 & 2,41 \\
neoplasia & 4 & 2,41 \\
usuário de drogas & 1 & 0,60 \\
\hline OUTRAS & 10 & 6,02 \\
TOTAL & 166 & 100 \\
\hline
\end{tabular}

o transplante renal supera a oferta. Como conseqüência, há um persi stente aumento do número de pacientes na lista de transplantes com maior tempo de espera, e cada vez mais idosos e doentes. ${ }^{6}$ Com a mel hora da qualidade do tratamento hemodialítico, existe a visão imediatista do candidato a transplante de trocar o "certo pelo duvidoso". V isão esta equivocada, uma vez que a sobrevida em longo prazo do paciente é maior. Com intuito de mudar esta situação, o serviço de diálise tem feito repetidas palestras instrutivas aos pacientes e seus familiares, além da divulgação de cartazes numa campanha denominada "R imdo à toa".

Entre as etiologias prevalentes em cada grupo, vale ressaltar influência do DM. Esta é a maior etiologia no grupo excluído da lista (30\%) e naquele em avaliação (42\%). No grupo dos pacientes aptos, sua presença é reduzida abruptamente $(5,5 \%)$, sendo a GNC (41\%), N H (12\%), N TIC (8\%) e NR (6,3\%), mais prevalentes. Esta iniqüidade de apresentação reflete a gravidade da doença do DM no estado geral do paciente, com grande repercussão em sua sobrevida, sendo um fator que reduz a possibilidade de inclusão em lista de transplante renal doador falecido, como já visto na literatura. ${ }^{7}$ Os pacientes portadores de DM tipo 1 do serviço de diálise estudado são habitual mente encaminhados ao serviço de transplante rim-pâncreas para inscrição em lista de espera, esperando que assim eles tenham menos tempo de exposição ao ambiente inflamado da DRC e DM. Mesmo sendo este centro dialítico analisado, um serviço com muitos pacientes provenientes da rede pública sem atendimento nefrológico prévio, existe, surpreendentemente, baixa incidência de causa indeterminada na etiologia primária. Vale ressaltar que a etiologia, classificada como glomerular ou intersticial, foi muitas vezes baseada em uma anamnese detalhada, sem diagnóstico histológico.

A pesar da mel hor sobrevida, a maior parte dos potenciais receptores nos EUA são incluídos na lista após seis meses de início de diálise, sendo este um dos fatores que aumenta o peso da DRC na saúde 
pública daquele país. 8 Para esta assertiva, deveria ser incluído no estudo, o tempo de tratamento dialítico antes da inclusão em lista. A avaliação para transplante renal deve ser iniciada antes do início da diálise, sendo fator preponderante para isto, o tempo em que o paciente foi encaminhado para o nefrologista e a adequada abordagem deste. ${ }^{10} 0$ custo desta avaliação e a manutenção de um paciente na lista de transplante após o início da diálise varia com a idade, reforçando a importância da precoce inscrição na lista de transplante renal. ${ }^{11} \mathrm{Um}$ trabal ho realizado no J apão demonstrou que o gasto com transplante renal no primeiro ano é em torno de 50000 dólares, e 19000 a partir do segundo. 0 custo da diálise é em torno de 46000 dólares por ano, sendo, portanto, o transplante renal mais barato a partir do segundo ano, como vários outros trabal hos confirmam.12

\section{Conclusão}

U m percentual esperado de pacientes desse centro encontra-se apto à inclusão em lista de espera de transplante renal doador falecido (30\% - Figura 1) e as maiores contra-indicações foram a idade avançada e a recusa à inscrição. A maior pendência para inclusão ou não em lista de transplante no grupo em investigação foi a cardiológica (46\%), avaliação esta obrigada pelo M G Transplante para todo paciente diabético maior que 45 anos. É de grande valia a publicação de mais trabalhos demonstrando situação de pacientes na lista de transplante doador falecido, com intuito, não de um consenso, mas de uma referência adaptada com a realidade populacional de cada centro de diálise.

Figura 1 - Situação dos pacientes na lista de espera para transplante renal - Fev/2005

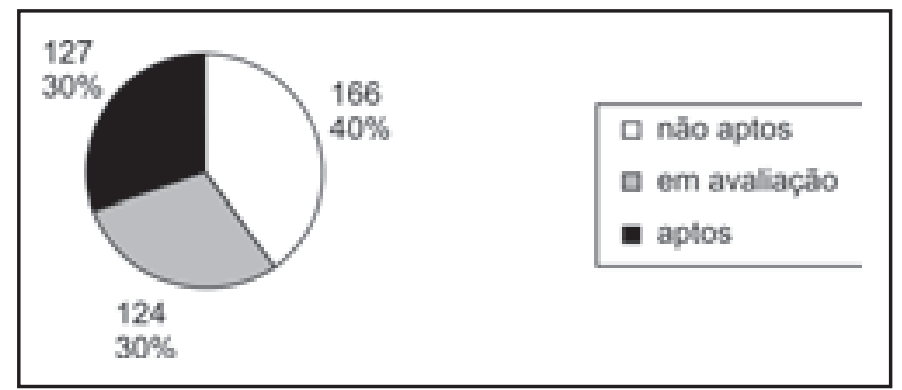

\section{ABSTRACT}

Introduction: The literature records no consensus as to the indications and counter-indications to the inclusion on the waiting list for deceased kidney donor transplantation in the replacement renal therapy center. Objectives: To assess the demographic profile, causes indicating the inclusion or not on that transplantation list, and the primary disease of patients under replacement renal therapy in that center. Methods: It was analyzed the database of patients under replacement renal therapy as to demographic data, primary disease and indication and counter-indications to the inclusion on the renal transplantation of deceased donor. Groups were divided in: $\mathrm{G} 1$ (A ble to Transplantation), G2 (U nable to transplant), G3 (A ssessment). Results: from 408 patients, G1 had 127 male patients (54\%): mean age of 43 years (15-65), 41\% with chronic glomerulonephritis, 12\% hypertensive nephrosclerosis, 10\% chronic interstitial tubulus nephritis, 6\% diabetes mellitus, 6\% reflux nephropathy. G2 had 166 patients: 46\% male (mean age: 62 years [25-88]), 30\% had diabetes mellitus, 15\% chronic glomerulonephritis, 14\% hypertensive nephrosclerosis, 11\% atheroembolic disease. G3 had 115 patients: $62 \%$ male (mean age of 49 years (16-65) , 42\% with diabetes mellitus, $4 \%$ atheroembolic disease, and $48 \%$ needing cardiologic assessment. The major reasons to the inclusion in the G 2 group were: age higher than 65 years and refusal to the transplantation. Conclusions: a percentage expected in that center was able to be included on the waiting list of deceased kidney donor (31\%), and the higher counter-indications were advanced age and ref usal.

Keywords: Dialysis, chronic renal failure, waiting list for kidney transplantation.

\section{REFERÊNCIAS}

1. Ohtake T, Kobayashi S, Moriya H, Maesato K, Saito S. High prevalence of occult coronary artery stenosis in patients with chronic kidney disease at the initiation of renal replacement therapy: An angiographic examination. J Am Soc Nephrol 2005; 16: 1141-1148

2. Fishbane S. Cardiovascular risk evaluation before kidney transplantation. J Am Soc Nephrol 2005; 16: 843-845

3. BRASIL - SBN. Censo da Sociedade Brasileira de Nefrologia Jan/2005. Disponível em "www.sbn.org.br" Acesso em 18/12/2006.

4. BRASIL - RBT. Registro Brasileiro de Transplantes Ano X - Ed.02. Disponível em www.abto.org.br. Acesso em 18/12/2006.

5. Zivcic-Cosic S, Fucak M, Orlic P, Vujaklija-Stipanovic K, Orlic L, Racki S, et al. Evaluation and selection of candidates for renal transplantation at the Clinical Hospital Center in Rijeka. Acta Med Croatica. 2003; 57(1): 65-68

6. Gallon LG, Leventhal JR, Kaufman DB. Pretransplant evaluation of renal transplant candidates. Semin Nephrol 2002; 22(6): 515-525
7. Oniscu GC, Schalkwijk AA, Johnson RJ, Brown H, Forsythe JL. Equity of access to renal transplant waiting list and renal transplantation in Scotland: cohort study. BMJ 2003; 327(7426):1261-1261

8. Epstein AM, Ayanian JZ, Keogh JH, Noonan SJ, Armistead N, Cleary PD, et al. Racial disparities in access to renal transplantation-clinically appropriate or due to underuse or overuse? N Engl J Med 2000; 343: 1537-1544

9. Owen WF Jr. Patterns of care for patients with chronic kidney disease in the United States: dying for improvement. J Am Soc Nephrol 2003; 14(2): S76-80.

10. Weng FL, Mange KC. A comparison of persons who present for preemptive and nonpreemptive kidney transplantation. Am J Kidney Dis 2003; 42(5): 1050-1057

11. Jeantet A, Piccoli GB, Malfi B, Messina M, Rossetti M, Tognarelli G, et al. Preparation of candidates for renal transplantation: cost analysis. Transplant Proc 2004; 36(3): 455-456

12. Nakajima I, Akamatsu M, Tojimbara T, Toma H, Fuchinque S. Economic study of renal transplantation: a single center. Transpl Proc 2001; 33: 1891-1892. 